\title{
The transcription factor KLF4 as an independent predictive marker for pathologic complete remission in breast cancer neoadjuvant chemotherapy: a case-control study
}

\author{
This article was published in the following Dove Press journal: \\ OncoTargets and Therapy \\ 24 October 2014 \\ Number of times this article has been viewed
}

\author{
Min Jun Dong' \\ Lin Bo Wang' \\ Zhi Nong Jiang ${ }^{2}$ \\ Mei Jin ${ }^{2}$ \\ Wen Xian $\mathrm{Hu}^{\prime}$ \\ Jian Guo Shen' \\ 'Department of Surgical Oncology, \\ ${ }^{2}$ Department of Pathology, Sir \\ Run Run Shaw Hospital, Zhejiang \\ University College of Medicine, \\ Hangzhou, People's Republic of China
}

\begin{abstract}
Background: To identify whether a stem cell biomarker, KLF4, may predict the pathologic tumor response to neoadjuvant chemotherapy for patients with locally advanced breast cancer.

Methods: Twelve locally advanced breast cancer patients who achieved pathologic complete remission (pCR) after neoadjuvant chemotherapy were identified and for each, three non-pCR breast cancer patients - matched for age, clinical tumor-node-metastasis stage, and neoadjuvant chemotherapy cycles - were selected. The relationship between KLF4 expression in the core needle biopsied cancer tissue and patient $\mathrm{pCR}$ rate was assessed using univariate and multivariate analysis.
\end{abstract}

Results: Receiver operating characteristic curve analysis showed that the patients with a histoscore of KLF4 expression $>0.18$ had a lower pCR rate. Multivariable analysis showed that higher KLF4 expression (odds ratio $0.013 ; 95 \%$ confidence interval $0.013-0.444 ; P=0.004$ ) was independently correlated with a lower pCR rate after neoadjuvant chemotherapy.

Conclusion: KLF4 overexpression was associated with lower pCR in locally advanced breast cancer patients undergoing neoadjuvant chemotherapy. This study suggests that KLF4 may serve as a predictor for $\mathrm{pCR}$ in patients with breast cancer after neoadjuvant chemotherapy.

Keywords: locally advanced breast cancer, predictor, stem cell biomarker, pathologic tumor response

\section{Introduction}

Breast cancer is a leading cause of morbidity and mortality among female cancer patients worldwide. ${ }^{1}$ Great improvements have been achieved in breast cancer treatment; studies and clinical practices on chemotherapy have contributed a lot and neoadjuvant chemotherapy has been proven to increase the radical excision rate and breast conservation rate, and those who achieved pathologic complete remission ( $\mathrm{pCR}$ ) gain a much better prognosis. However, in less responsive cases, there may be risk in delaying surgery. The National Surgical Adjuvant Breast and Bowel Project B-18 trial revealed that only $13 \%$ of the breast cancer patients who achieved pCR could achieve better overall survival, while $20 \%$ of the patients who had no response to neoadjuvant chemotherapy may have led to worse survival. ${ }^{2}$ These results make it important to find out whether there are some special hallmarks that may predict the response to neoadjuvant chemotherapy in locally advanced breast cancer patients.
Correspondence: Jian Guo Shen Department of Surgical Oncology, Sir Run Run Shaw Hospital, Zhejiang University College of Medicine, 3 Qingchun East Road, Jianggan, Hangzhou 310016, People's

Republic of China

Fax +86 57। 86044817

Email drshenjianguo@I63.com 
Some reports have suggested that a few molecular markers, such as ER or HER2, may be related to the therapeutic response; however, the clinical usefulness of these markers remains uncertain or controversial. ${ }^{3,4}$ Thus, identifying factors that can predict the efficacy of neoadjuvant chemotherapy is essential for decision making in the management of patients with breast cancer.

It is well known that the efficacy of chemotherapy for malignant tumors differs a lot, but the exact reasons that may impact the effect of anticancer drugs remain unclear. A growing number of reports show that a higher proportion of cancer stem cells (CSCs) correlates with an increased incidence of relapse due to chemotherapy-resistant outgrowth and worse overall survival. ${ }^{5}$ In addition, resistant CSCs have the ability to reinitiate disease either immediately after treatment or after significant dormancy. ${ }^{6}$

In recent years, it has been reported that the transcription factor KLF4 and three other transcription factors Oct4, c-Myc, and Sox 2 - are capable of reprogramming fibroblasts into induced pluripotent stem cells, hinting that KLF4 is indispensable for the maintenance of stem cells. ${ }^{7.8}$ Furthermore, forced expression of KLF4 has demonstrated a role in self-renewal. ${ }^{9,10}$ Studies have also shown that KLF4 is essential for maintaining CSCs and for promoting migration and invasion, resulting in tumor formation in vivo. ${ }^{11}$ However, whether KLF4 may influence the tumor response to chemotherapy has not been studied. The present study sought to identify whether transcription factor KLF4 can predict pathologic tumor response to neoadjuvant chemotherapy in locally advanced breast cancer.

\section{Materials and methods}

\section{Study design}

Approval to utilize the authors' institution's breast cancer database and tissue bank was obtained from the institutional internal review board. From June 2002 to December 2010, 1,135 patients with breast cancer underwent surgery at the Department of Surgical Oncology, Sir Run Run Shaw Hospital, Zhejiang University College of Medicine (Hangzhou, People's Republic of China). Among them, 318 patients underwent neoadjuvant chemotherapy followed by surgery, and among the patients who had achieved pCR after three to four cycles of neoadjuvant chemotherapy, only 12 patients' remaining core needle biopsy tissue was available for immunohistochemical (IHC) staining in the study. For each of the 12 patients, three breast cancer patients who did not achieve pCR were selected as controls and were matched according to age ( \pm 2 years), clinical tumor-node-metastasis stage, and neoadjuvant chemotherapy cycles. Therefore, a total of 48 patients were enrolled in the present study.

All of the 48 patients were pathologically diagnosed through core needle biopsy; the clinical stage was evaluated using the seventh edition Union for International Cancer Control/ American Joint Committee on Cancer system. These patients received three or four cycles of neoadjuvant chemotherapy, and none of them underwent neoadjuvant endocrine therapy and/or radiation therapy. Thirty-nine patients (83.3\%) had undergone a modified radical mastectomy, while nine patients (16.7\%) received breast conservation therapy (lumpectomy with tumor-free margin, axillary lymph node dissection, and breast radiation). After surgery, the patients continued to complete the chemotherapy regimens as planned.

\section{IHC}

IHC staining for KLF4 expression was performed on formalin-fixed, paraffin-embedded sections of core needle biopsy specimen. The slides were deparaffinized in xylene and rehydrated in gradient ethanol solutions. Endogenous peroxidase was blocked with $0.3 \% \mathrm{H}_{2} \mathrm{O}_{2}$ in methanol for 5 minutes. The slides were immersed in $10 \mathrm{mM}$ citric buffer (pH 6.0) with heating for 15 minutes for antigen retrieval. Nonspecific binding was blocked by preincubation with $10 \%$ fetal calf serum in phosphate-buffered saline with $0.01 \%$ sodium azide, and the slides were incubated in a humid chamber for 1 hour with polyclonal rabbit anti-KLF4 antibody (ab72543; Abcam plc, Cambridge, UK). After washing three times in phosphate-buffered saline, the slides were incubated with the EnVision ${ }^{\mathrm{TM}}$ horseradish peroxidase complex (undiluted; Agilent Technologies, Santa Clara, CA, USA) for 60 minutes. The slides were visualized with diaminobenzidine (Agilent) and then counterstained with hematoxylin, dehydrated, cleared, and mounted with resinous mounting medium. The positive control was skin tissue that was known to exhibit high expression levels of KLF4. The negative control was the same skin tissue, with phosphatebuffered saline replacing the KLF4 antibody during the IHC staining. Most of the biopsy samples contained some adjacent noncancerous tissue, though not a lot, and the IHC staining in the study found that all of the noncancerous tissue had low/negative KLF4 expression.

\section{$\mathrm{IHC}$ analysis}

The immunoreactivity of KLF4 was assessed according to the intensity and distribution of cell cytoplasm staining by diaminobenzidine chromogen. Cell cytoplasm 
staining intensity was scored using a weighted histoscore method. ${ }^{12}$ The intensity of staining was graded as $3+$ (dark brown; strongly positive), $2+$ (brown; moderately positive), $1+$ (light brown; weekly positive), and negative. The final histoscores were calculated as follows: $(1 \times$ percentage of cells staining light brown $)+(2 \times$ percentage of cells staining brown $)+(3 \times$ percentage of cells staining dark brown $)$.

Scoring was undertaken by two independent pathologists who did not know the clinical outcomes. Discrepant scoring was reevaluated by the two pathologists and agreement was reached after appropriate discussion.

\section{Assessment of pathologic response}

After surgery, all tumor specimens were examined by the pathologists. Patients with pCR were defined as those who had noninvasive or in situ cancer in the breast specimen as well as those in whom no residual cancer was identified, regardless of the status of the axillary lymph node. ${ }^{13}$

\section{Follow-up}

Adjuvant sequential systemic chemotherapy, conserved breast and/or axillary irradiation, and endocrine therapy were accordingly offered and administered as indicated per current standard of care. Surveillance protocol consisted of a history and physical examination every 3 months for 3 years, every 6 months in the following 2 years, and annually thereafter. A complete physical examination, chest $\mathrm{X}$-ray, mammogram, annual mammogram, and complete blood workup were performed for each patient. Additional imaging, including computed tomography, positron emission tomography, and magnetic resonance imaging were performed when indicated. All of the patients were followed up until October 2013. The median follow-up period was 55.7 months. During the follow-up, 34 patients were still alive and 14 patients had died.

\section{Statistical analysis}

All statistical calculations were carried out using SPSS 16.0 for Windows ${ }^{\circledR}$ (SPSS, Inc., Chicago, IL, USA). Chi-square or Fisher's exact tests were used to determine the significance of associations between pathologic findings and categoric variables. The median age was analyzed by the independent samples $t$-test. A receiver operating characteristic curve was constructed to define the cutoff value for the various continuous variables that were correlated with predicting pCR. Logistic regression analysis was used to identify correlations between pathologic response and continuous variables. Multivariate analyses were used to identify correlations between pathologic response and all potential parameters involved using a binary logistic regression model. The Kaplan-Meier survival method and the log-rank test were used to generate and compare survival curves. Multivariate analyses were performed using the Cox proportional hazard regression models. Risk ratios and $95 \%$ confidence intervals were calculated from the model. $P<0.05$ was considered to indicate a significant difference.

\section{Results \\ PCR after neoadjuvant chemotherapy predicts better survival}

Twelve patients who achieved pCR through neoadjuvant chemotherapy and 36 patients who did not achieve pCR were studied. The age, clinical tumor-node-metastasis stage, and neoadjuvant chemotherapy cycles were well matched, and there were no significant differences in the major clinical and pathologic parameters between the two groups (Table 1).

Overall survival in the pCR group and the non-pCR group was compared, with a median follow-up period of 55.7 months. The 5-year survival rate was significantly higher in the pCR group (91.7\%) than in the non-pCR group $(61.1 \%$; $P=0.034$ ) (Figure 1).

\section{High KLF4 expression in breast cancer predicts lower $\mathrm{PCR}$ rate}

The average KLF4 expression histoscore was 1.02, with a range of $0-2.70$ (Figure 2). The pCR group had a lower KLF4 histoscore of $0.24 \pm 0.49$, and the non-pCR group had a higher KLF4 histoscore of $1.28 \pm 0.94(P=0.001)$.

Using receiver operating characteristic analysis, a KLF4 histoscore cutoff level of 0.18 was found. The sensitivity and specificity of this cutoff value in predicting tumor pCR was $75.0 \%$ and $83.3 \%$, respectively. Consequently, the correlation between KLF4 histoscores $(\geq 0.18$ and $<0.18)$ and pCR rate was investigated by chi-square statistical analysis. It was found that the high KLF4 expression group had a lower pCR rate $(P<0.001$; Table 2$)$.

In multivariate analysis, while controlling for other variables studied in univariate analysis, KLF4 expression was found to be independently correlated with $\mathrm{pCR}$ (odds ratio $0.013 ; 95 \%$ confidence interval 0.013-0.444; $P=0.004$ ) (Table 3).

\section{High KLF4 expression predicts worse outcome}

The median survival duration in patients who had a tumor with low or high KLF4 expression was 78 months and 47 months, respectively. Elevated KLF4 expression was 
Table I Analysis of various factors that may influence the outcome of neoadjuvant chemotherapy (chi-square test)

\begin{tabular}{|c|c|c|c|}
\hline & $\begin{array}{l}\mathrm{PCR} \\
(\mathrm{n}=12)\end{array}$ & $\begin{array}{l}\text { non-pCR } \\
(n=36)\end{array}$ & $P$-value \\
\hline Age & $47.8 \pm 6.8$ & $47.5 \pm 9.4$ & 1.0 \\
\hline AJCC cTNM stage & & & 1.0 \\
\hline II & 7 & 21 & \\
\hline III & 5 & 15 & \\
\hline Neoadjuvant chemotherapy cycles & & & 1.0 \\
\hline Three cycles & 7 & 21 & \\
\hline Four cycles & 5 & 15 & \\
\hline ER & & & 0.302 \\
\hline Positive ( $\geq 1 \%)$ & 3 & 15 & \\
\hline Negative $(<1 \%)$ & 9 & 21 & \\
\hline $\mathrm{PR}$ & & & 0.499 \\
\hline Positive ( $\geq 10 \%)$ & 4 & 18 & \\
\hline Negative $(<10 \%)$ & 8 & 18 & \\
\hline HER2 & & & $0.44 I$ \\
\hline Positive & 2 & 10 & \\
\hline Negative & 10 & 26 & \\
\hline Ki67 & & & 0.316 \\
\hline Positive ( $\geq 14 \%)$ & 8 & 20 & \\
\hline Negative $(<\mid 4 \%)$ & 4 & 16 & \\
\hline $\mathrm{cN}$ stage & & & 0.309 \\
\hline 0 & 9 & 16 & \\
\hline I & 2 & 13 & \\
\hline 2 & I & 5 & \\
\hline 3 & 0 & 2 & \\
\hline Tumor grade & & & 0.399 \\
\hline I & 3 & 6 & \\
\hline 2 & 3 & 17 & \\
\hline 3 & 6 & 13 & \\
\hline $\begin{array}{l}\text { Neoadjuvant chemotherapy } \\
\text { regimen }\end{array}$ & & & 0.104 \\
\hline Anthracyclines based & 9 & 15 & \\
\hline $\begin{array}{l}\text { Anthracyclines } \\
\text { combined with taxane }\end{array}$ & 3 & 16 & \\
\hline Others & 0 & 5 & \\
\hline Operation procedure & & & 0.135 \\
\hline Mastectomy & 8 & 31 & \\
\hline Breast-conserving surgery & 4 & 5 & \\
\hline
\end{tabular}

Abbreviations: AJCC, American Joint Committee on Cancer; $\mathrm{cN}$, clinical node; cTNM, clinical tumor-node-metastasis; pCR, pathologic complete remission.

associated with inferior disease-free survival $(P=0.044$; Figure 3A), but KLF4 expression did not significantly contribute to overall survival $(P=0.419$; Figure $3 \mathrm{~B})$.

\section{Discussion}

Understanding tumor biology is essential for early diagnosis of cancers, decision making for cancer treatment strategies, and developing target specific cancer therapies. There is evidence to suggest that transcription factor KLF4 plays a crucial role in the progression of breast carcinoma, ${ }^{14}$ squamous cell carcinoma, ${ }^{15}$ lung cancer, ${ }^{16}$ and colon cancer. ${ }^{13}$

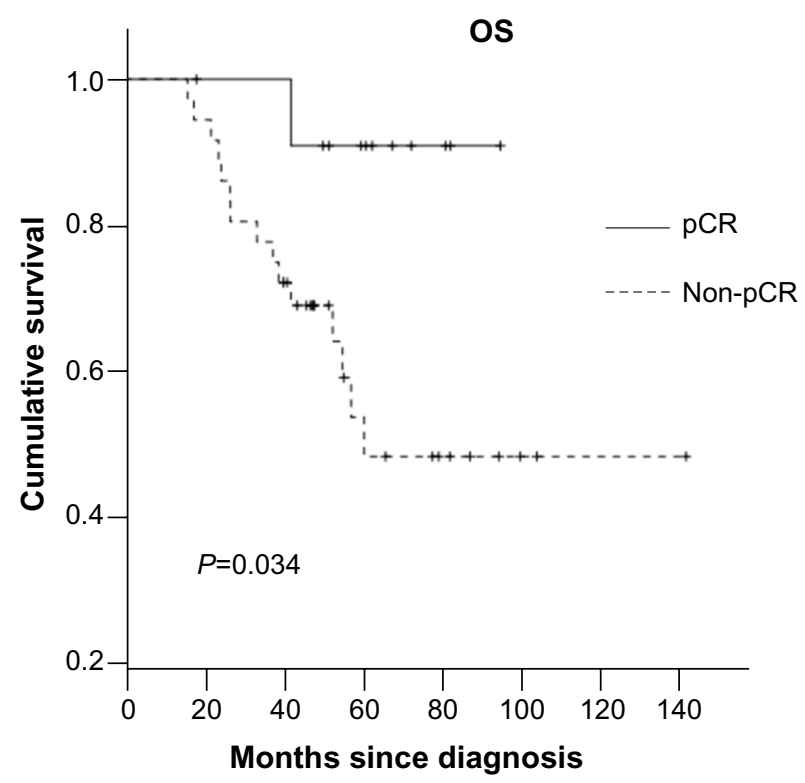

Figure I Kaplan-Meier survival analysis for locally advanced breast cancer patients who received neoadjuvant chemotherapy according to different pathologic response. Abbreviations: OS, overall survival; $\mathrm{PCR}$, pathologic complete remission.

Therefore, KLF4 is a potential diagnostic marker, prognostic factor, or target for novel therapy.

To the authors' knowledge, this is the first study to investigate whether KLF4 is a predictor for breast cancer response to neoadjuvant chemotherapy. The results show that the clinical and pathologic features of the pCR patients appeared to be similar to the non-pCR controls. The 5-year survival rate was significantly higher in the $\mathrm{pCR}$ group, and this result is consistent with that of the National Surgical Adjuvant Breast and Bowel Project B-18 trial. ${ }^{2}$ Receiver operating characteristic curve analysis showed that the histoscore of KLF4 expression, with 0.18 as the cutoff value, could best differentiate between pCR and non-pCR. In multivariate analysis, high KLF4 expression was found to be independently correlated with a lower pCR rate.

It is well known that more cycles of neoadjuvant chemotherapy and the addition of docetaxel to anthracyclines could significantly increase pathologic response rates in both ER-positive and ER-negative breast cancer patients. ${ }^{17,18}$ In the present study, the impact of these two important factors was eliminated, so KLF4 was demonstrated as an independent predictor for $\mathrm{pCR}$.

It has been widely confirmed that CSCs play an important role in resistance to conventional chemotherapies compared with the more differentiated cells in the non-CSC compartment. There have been several studies implicating CSCs as being chemotherapy resistant in a variety of 

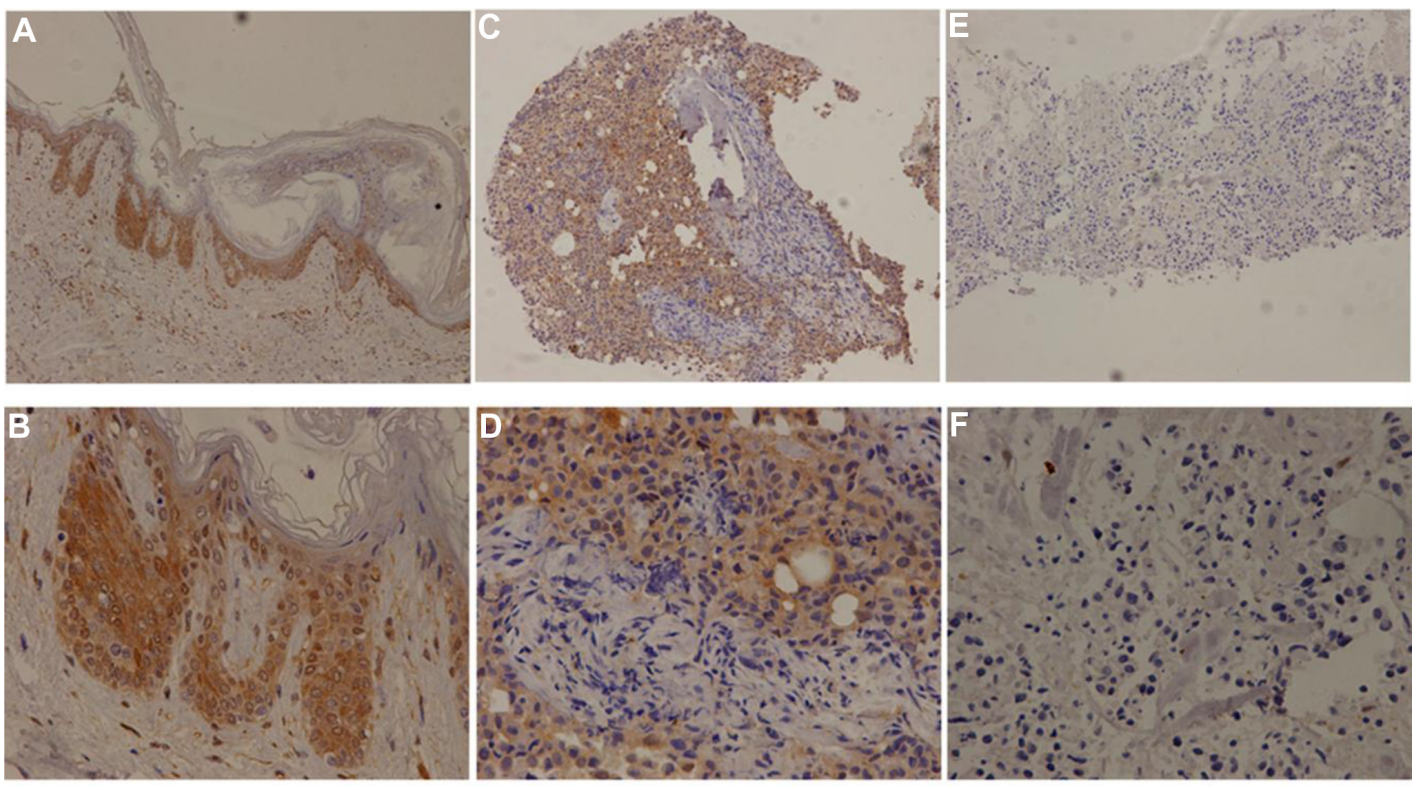

Figure 2 Immunohistochemical KLF4 staining in normal skin tissue (A, B) and in primary biopsy tissues of human breast cancer (C-F).

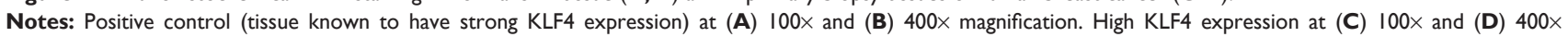
magnification. Low KLF4 expression at (E) 100× and (F) 400× magnification.

different cancers, such as breast cancer, ${ }^{19}$ pancreatic cancer, ${ }^{20}$ hepatocellular carcinoma, ${ }^{21}$ and colon cancer. ${ }^{22}$

One possible reason why KLF4 overexpression was an independent predictor for lower $\mathrm{pCR}$ rate might be related to its contribution in increasing breast CSCs. KLF4 has been widely demonstrated to be necessary for the reprogramming of differentiated cells into pluripotent stem cells and the maintenance of breast CSCs. ${ }^{6}$ Furthermore, KLF4 promotes self-renewal of CSCs and may increase the risk of distant metastasis. ${ }^{23}$ In vitro studies demonstrated that KLF4 knockdown significantly delayed tumor development of breast cancer cells in a xenograft mouse model. In addition, downregulation of KLF4 significantly decreased CSC-enriched populations by using several different CSC markers (eg, CD44+/CD24-, ESA+/PROCR+). Knockdown of KLF4 decreased tumorigenesis in NOD/SCID mice, supporting an important role of KLF4 in the maintenance of breast CSCs. ${ }^{11}$

There are a few contradictory studies debating whether some other factors may have the ability to predict the tumor response to chemotherapy in breast cancer.

Table 2 Chi-square statistical analysis to identify KLF4 expression and pathologic complete remission

\begin{tabular}{llll}
\hline & pCR & non-pCR & P-value \\
\hline KLF4 high $(\geq 0.18)$ & $2(16.7 \%)$ & $27(75 \%)$ & $<0.001$ \\
KLF4 low $(<0.18)$ & $10(83.3 \%)$ & $9(25 \%)$ & \\
\hline
\end{tabular}

Abbreviation: $\mathrm{PCR}$, pathologic complete remission.
Retrospective studies by $\mathrm{Li}$ et $\mathrm{al}^{24}$ and Colleoni et $\mathrm{al}^{25}$ reviewed locally advanced breast cancer patients who had received neoadjuvant chemotherapy with docetaxel and/or epirubicin. They found that ER/PR status (both negative), but not HER2 or Ki67, were independent predictive factors of pCR to neoadjuvant chemotherapy. However, an earlier retrospective study indicated that ER-rich patients had a significantly higher response rate to chemotherapy, ${ }^{26}$ and another retrospective study by Tiezzi et al suggested that IHC phenotypes were not able to predict response to neoadjuvant chemotherapy with docetaxel plus epirubicin. ${ }^{4}$ In the present study, no significant differences in ER, PR, HER2, and Ki67 expression were found between the pCR group and the non-pCR group. ER/PR status has an established role in determining the tumor response to hormonal therapy, but its role in predicting response to chemotherapy is less clear.

Table 3 Multivariate logistic analysis to identify predictors of pathologic complete remission in locally advanced breast cancer patients with neoadjuvant chemotherapy

\begin{tabular}{llll}
\hline & OR & $\mathbf{9 5 \%} \mathbf{C l}$ & P-value \\
\hline KLF4 $(\geq 0.18$ versus $<0.18)$ & 0.077 & $0.014-0.415$ & 0.003 \\
ER (positive versus negative) & 1.317 & $0.159-10.900$ & 0.798 \\
PR (negative versus positive) & 0.430 & $0.066-2.817$ & 0.379 \\
HER2 (positive versus negative) & 0.278 & $0.038-2.028$ & 0.207 \\
Ki67 ( $\geq 14 \%$ versus $<14 \%)$ & 2.853 & $0.497-16.361$ & 0.239 \\
\hline
\end{tabular}

Abbreviations: $\mathrm{Cl}$, confidence interval; $\mathrm{OR}$, odds ratio; $\mathrm{PCR}$, pathologic complete remission. 

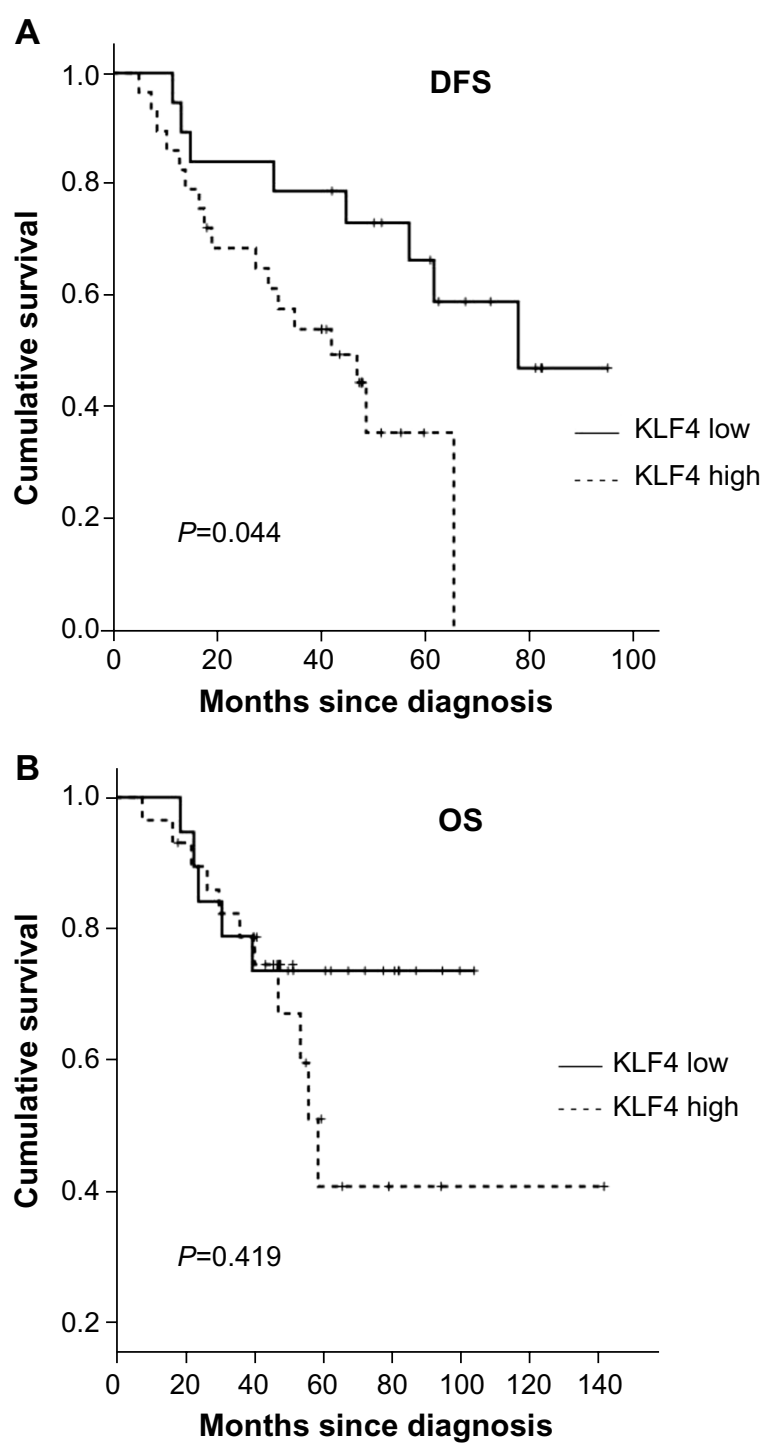

Figure 3 Prognostic disease-free survival and overall survival analyses for locally advanced breast cancer patients who received neoadjuvant chemotherapy according to KLF4 expression.

Notes: (A) Disease-free survival (DFS). (B) Overall survival (OS).

Furthermore, the value of KLF4 in predicting breast cancer survival was observed in the present study. Elevated KLF4 expression resulted in worse disease-free survival, but no significant impact on overall survival was indicated. This may be because the follow-up time was not long enough. The fact that KLF4 overexpression resulted in worse survival has been demonstrated by many clinical data, and the reason has been widely discussed. Some recent studies suggested that KLF4 participates in many signaling pathways that support multiple aggressive traits in breast cancer, such as maintenance of high glycolytic metabolism ${ }^{27}$ and upregulation of Notch signaling. ${ }^{11,28}$ The present study indicates that high KLF4 expression leads to a worse outcome possibly due to resistance of chemotherapy.
KLF4's utility may not be in its use as a single predictor, but rather as part of a larger array of molecular markers for predicting response to neoadjuvant chemotherapy for locally advanced breast cancer. The authors plan to study the potential mechanism of how KLF4 influences the response of breast cancer cells to chemotherapy in vitro, with the aim of finding some targets with the aim of finding some therapeutic targets to improve the efficacy of chemotherapy.

\section{Conclusion}

This study was the first to identify that breast cancer patients with low KLF4 expression rather than high expression benefit more from neoadjuvant chemotherapy, and suggests that KLF4 may serve as a predictive biomarker for $\mathrm{pCR}$ in patients with locally advanced breast cancer after neoadjuvant chemotherapy. The findings from these case-control retrospective data indicate the need for a larger, prospective clinical trial.

\section{Acknowledgments}

This study was supported by the Program for Innovative Research Team in Zhejiang Province (grant number: 2010R50046).

\section{Disclosure}

The authors report no conflicts of interest in this work.

\section{References}

1. Siegel R, Naishadham D, Jemal A. Cancer statistics, 2013. CA Cancer J Clin. 2013;63(1):11-30.

2. Fisher B, Brown A, Mamounas E, et al. Effect of preoperative chemotherapy on local-regional disease in women with operable breast cancer: findings from National Surgical Adjuvant Breast and Bowel Project B-18. J Clin Oncol. 1997;15(7):2483-2493.

3. Li XR, Liu M, Zhang YJ, et al. Evaluation of ER, PgR, HER-2, Ki-67, cyclin $\mathrm{D} 1$, and $\mathrm{nm} 23-\mathrm{H} 1$ as predictors of pathological complete response to neoadjuvant chemotherapy for locally advanced breast cancer. Med Oncol. 2011;28(Suppl 1):S31-S38.

4. Tiezzi DG, Andrade JM, Ribeiro-Silva A, Zola FE, Marana HR, Tiezzi MG. HER-2, p53, p21 and hormonal receptors proteins expression as predictive factors of response and prognosis in locally advanced breast cancer treated with neoadjuvant docetaxel plus epirubicin combination. BMC Cancer. 2007;7:36

5. van Rhenen A, Feller N, Kelder A, et al. High stem cell frequency in acute myeloid leukemia at diagnosis predicts high minimal residual disease and poor survival. Clin Cancer Res. 2005;11(18):6520-6527.

6. Klein CA, Holzel D. Systemic cancer progression and tumor dormancy: mathematical models meet single cell genomics. Cell Cycle. 2006;5(16):1788-1798.

7. Takahashi K, Yamanaka S. Induction of pluripotent stem cells from mouse embryonic and adult fibroblast cultures by defined factors. Cell. 2006;126(4):663-676.

8. Wernig M, Meissner A, Foreman R, et al. In vitro reprogramming of fibroblasts into a pluripotent ES-cell-like state. Nature. 2007;448(7151):318-324. 
9. Bruce SJ, Gardiner BB, Burke LJ, Gongora MM, Grimmond SM, Perkins AC. Dynamic transcription programs during ES cell differentiation towards mesoderm in serum versus serum-freeBMP4 culture. BMC Genomics. 2007;8:365.

10. Zhang P, Andrianakos R, Yang Y, Liu C, Lu W. Kruppel-like factor 4 (KLF4) prevents embryonic stem (ES) cell differentiation by regulating Nanog gene expression. J Biol Chem. 2010;285(12):9180-9189.

11. Yu F, Li J, Chen H, et al. Kruppel-like factor 4 (KLF4) is required for maintenance of breast cancer stem cells and for cell migration and invasion. Oncogene. 2011;30(18):2161-2172.

12. Teo K, Gemmell L, Mukherjee R, Traynor P, Edwards J. Bad expression influences time to androgen escape in prostate cancer. BJU Int. 2007;100(3):691-696.

13. Dang DT, Chen X, Feng J, Torbenson M, Dang LH, Yang VW. Overexpression of Kruppel-like factor 4 in the human colon cancer cell line RKO leads to reduced tumorigenecity. Oncogene. 2003;22(22):3424-3430

14. Foster KW, Frost AR, McKie-Bell P, et al. Increase of GKLF messenger RNA and protein expression during progression of breast cancer. Cancer Res. 2000;60(22):6488-6495.

15. Foster KW, Liu Z, Nail CD, et al. Induction of KLF4 in basal keratinocytes blocks the proliferation-differentiation switch and initiates squamous epithelial dysplasia. Oncogene. 2005;24(9):1491-1500.

16. $\mathrm{Hu}$ W, Hofstetter WL, Li H, et al. Putative tumor-suppressive function of Kruppel-like factor 4 in primary lung carcinoma. Clin Cancer Res. 2009;15(18):5688-5695.

17. Andrade JM, Carrara HH, Pimentel FF, et al. Taxane-based chemotherapy enhances response to neoadjuvant treatment for stage II and III breast cancer. Med Oncol. 2011;28(Supp1 1):S65-S69.

18. Bear HD, Anderson S, Brown A, et al. The effect on tumor response of adding sequential preoperative docetaxel to preoperative doxorubicin and cyclophosphamide: preliminary results from National Surgical Adjuvant Breast and Bowel Project Protocol B-27. J Clin Oncol. 2003;21(22):4165-4174.
19. Charafe-Jauffret E, Monville F, Ginestier C, Dontu G, Birnbaum D, Wicha MS. Cancer stem cells in breast: current opinion and future challenges. Pathobiology. 2008;75(2):75-84.

20. Hermann PC, Huber SL, Herrler T, et al. Distinct populations of cancer stem cells determine tumor growth and metastatic activity in human pancreatic cancer. Cell Stem Cell. 2007;1(3):313-323.

21. Ma S, Lee T, Zheng BJ, Chan KW, Guan XY. CD133+ HCC cancer stem cells confer chemoresistance by preferential expression of the Akt/PKB survival pathway. Oncogene. 2008;27(12):1749-1758.

22. Todaro M, Alea MP, Di Stefano AB, et al. Colon cancer stem cells dictate tumor growth and resist cell death by production of interleukin-4. Cell Stem Cell. 2007;1(4):389-402.

23. Okuda H, Xing F, Pandey PR, et al. miR-7 suppresses brain metastasis of breast cancer stem-like cells by modulating KLF4. Cancer Res 2013;73(4):1434-1444.

24. Li XR, Liu M, Zhang YJ, et al. ER, PgR, HER-2, Ki-67, topoisomerase $\mathrm{II} \alpha$, and $\mathrm{nm} 23-\mathrm{H} 1$ proteins expression as predictors of pathological complete response to neoadjuvant chemotherapy for locally advanced breast cancer. Med Oncol. 2011;28(Suppl 1):S48-S54.

25. Colleoni M, Viale G, Zahrieh D, et al. Expression of ER, PgR, HER1, HER2, and response: a study of preoperative chemotherapy. Ann Oncol. 2008;19(3):465-472.

26. Kiang DT, Frenning DH, GoldmanAI, Ascensao VF, Kennedy BJ. Estrogen receptors and responses to chemotherapy and hormonal therapy in advanced breast cancer. $N$ Engl J Med. 1978;299(24):1330-1334.

27. Moon JS, Kim HE, Koh E, et al. Kruppel-like factor 4 (KLF4) activates the transcription of the gene for the platelet isoform of phosphofructokinase (PFKP) in breast cancer. J Biol Chem. 2011;286(27):23808-23816.

28. Liu Z, Teng L, Bailey SK, et al. Epithelial transformation by KLF4 requires Notch1 but not canonical Notch1 signaling. Cancer Biol Ther. 2009;8(19):1840-1851.
OncoTargets and Therapy

\section{Publish your work in this journal}

OncoTargets and Therapy is an international, peer-reviewed, open access journal focusing on the pathological basis of all cancers, potential targets for therapy and treatment protocols employed to improve the management of cancer patients. The journal also focuses on the impact of management programs and new therapeutic agents and

\section{Dovepress}

protocols on patient perspectives such as quality of life, adherence and satisfaction. The manuscript management system is completely online and includes a very quick and fair peer-review system, which is all easy to use. Visit http://www.dovepress.com/testimonials.php to read real quotes from published authors. 